\title{
Thematic Continuities and Fresh Perspectives
}

\author{
Christopher Coenen
}

Published online: 13 July 2016

C) Springer Science+Business Media Dordrecht 2016

The current issue of NanoEthics. Studies of New and Emerging Technologies starts with an illuminating analysis of the relevance of informed consent concerning the risks posed by engineered nanomaterials. Shannon Lydia Spruit, Ibo van de Poel and Neelke Doorn concede that we can learn from experiences of informed consent in treatment and research contexts but argue that we should be aware that informed consent traditionally pertains to certain aspects of the relationships between doctors and patients and between researchers and research participants rather than to the relationships between producers and consumers or between employers and employees, which are of greater relevance in the case of engineered nanomaterials. The authors analyse three major relational factors that influence whether valid informed consent is obtainable: dependency, personal proximity and the existence of shared interests. In the conclusions, they draw from their highly original analysis; Spruit and her colleagues suggest that a constructive yet speculative approach to informed consent would conceive these three relational aspects as communicating vessels.

In her thorough analysis of the pressing and muchdiscussed topic of CRISPR/Cas9 technology, Martina Baumann first takes a brief look at the arguments put forward by different stakeholders with a view to

C. Coenen $(\bowtie)$

Institute for Technology Assessment and Systems Analysis (ITAS), Karlsruhe Institute of Technology (KIT), POB 3640, 76021 Karlsruhe, Germany

e-mail: christopher.coenen@kit.edu identifying the different ways in which they deal with the topic and to pointing out any shortcomings of these various perspectives. Based on an analysis of both the technoscientific state of the art and the ethical, legal and social aspects of CRISPR/Cas9 germline editing, she draws up a scenario that illustrates the future use of CRISPR/Cas9 in humans, including relevant ethical questions and regulatory challenges. The author outlines an agenda for the ethics of CRISPR/Cas9 genome editing in humans and for strongly interdisciplinary collaboration in particular.

In their book reviews, Michael G. Sherbert and Nora S. Vaage each give their appraisals of an edited volume on the topics of 'human enhancement' and transhumanism, thereby continuing a long tradition of this journal's coverage of these still highly controversial issues. Recently, ideas and concerns about the potential offered by CRISPR/CAS9 germline editing for the purposes of 'human enhancement' have attracted significant academic and broader public attention. The two reviewed collections of essays are testament to the increasing diversity and depth of academic discourse on visions of enhanced human beings.

I am particularly pleased that the present issue also includes a special section on responsible research and innovation (RRI; cf. [1, 2]), guest-edited by Stephan Lingner and by our journal's founding editor John Weckert. As regards the analyses and discussions in this special section, I refer you to the introduction by the guest editors and to the papers themselves, which were written by Doris Schroeder, Sally Dalton-Brown, Benjamin Schrempf and David Kaplan, by Sarah Laird and 
Rachel Paula Wynberg, by Kristin Hagen and by John Weckert, Hector Rodriguez Valdes and Sadjad Soltanzadeh.

There are only two things I wish to point out regarding this excellent special section: firstly, it quite prominently features the topic of synthetic biology, and as you will see from our 'Online First' section, more papers on this subject will be published in our December issue. This perpetuates our journal's custom of publishing important contributions to discourse on this emerging field of technoscience. Secondly, this special section features an article by the journal's founding editor and his co-authors in which they explore a question that is crucially relevant not only to RRI in a global context but also more generally to globalisation: namely how to deal with the widespread argument 'If we don't somebody else will'. I look forward very much to the discussions that the articles in this special section will inspire with regard to RRI and hope you will find the present issue insightful and pleasant reading.

\section{References}

1. Owen R, Bessant JR, Heintz M (eds) Responsible innovation: managing the responsible emergence of science and innovation in society. Wiley, Chichester

2. Coenen C (2016) Broadening discourse on Responsible Research and Innovation (RRI). NanoEthics 10(1):1-4 